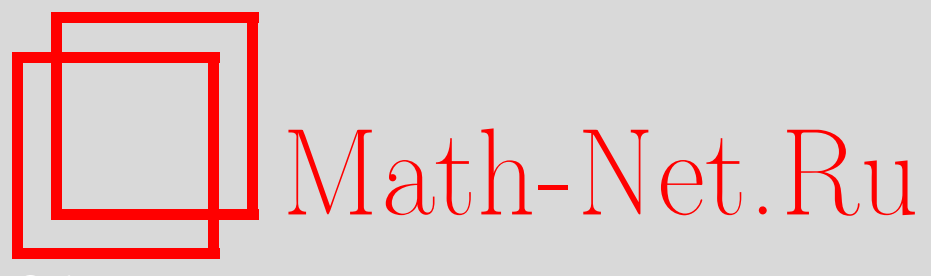

В. А. Киосак, В. С. Матвеев, Й. Микеш, И. Г. Шандра, О степени геодезической подвижности римановых метрик, Матем. заметки, 2010, том 87, выпуск 4, 628-629

DOI: https://doi.org/10.4213/mzm6605

Использование Общероссийского математического портала Math-Net.Ru подразумевает, что вы прочитали и согласны с пользовательским соглашением http://www.mathnet.ru/rus/agreement

Параметры загрузки:

IP : 54.172 .240 .79

26 апреля 2023 г., 13:39:07

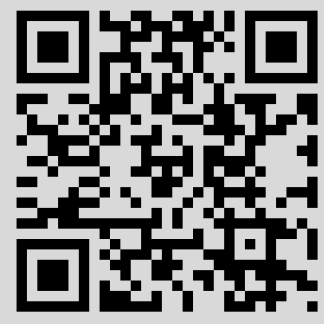




\section{О степени геодезической подвижности римановых метрик}

\section{В. А. Киосак, В. С. Матвеев, Й. Микеш, И. Г. Шандра}

Две метрики $g$ и $\bar{g}$ на одном многообразии $M$ называются геодезически эквивалентными, если каждая геодезическая метрики $g$, после подходящей параметризации, является геодезической $\bar{g}$. Известно [1], что геодезически эквивалентные метрики находятся в однозначном соответствии с невырожденными симметрическими $(0,2)$ тензорами $a_{i j}$, удовлетворяющими уравнению

$$
a_{i j, k}=\frac{1}{2}\left(a_{\alpha, i}^{\alpha} g_{j k}+a_{\alpha, j}^{\alpha} g_{i k}\right),
$$

где $a_{\alpha}^{\alpha}=a_{\alpha \beta} g^{\alpha \beta}, g^{i j}$ - элементы обратной матрицы к $g_{i j}$, запятой обозначена ковариантная производная по связности $(M, g)$. Эти уравнения линейны, множество их решений образует линейное векторное пространство, его размерность называется степенъю геодезической подвижности метрики $g$ и обозначается $p ; p$ - является числовой характеристикой мощности геодезического класса данной метрики $g_{i j}$ (см. [1]).

Давно замечено [2], что метрики с большой степенью подвижности допускают конциркулярные ковекторные поля. Напомним, что ковекторное поле $\varphi_{i}$ на $(M, g)$ называется конциркулярным, если

$$
\varphi_{i, j}=\rho g_{i j}
$$

для некоторой функции $\rho$ на многообразии $(M, g)$.

Линейное пространство конциркулярных векторных полей для метрики $g_{i j}$ будем обозначать $\operatorname{Con}(M)$.

Следующее утверждение было сформулировано в работе [3].

Теорема 1. Степень геодезической подвижности $n$-мерного $(n \geqslant 3)$ риманова (с положительно определенной метрикой) пространства $(M, g)$, отличного от пространства постоянной кривизны, может принимать лишь значения $p=m(m+1) / 2+l$, где $m=\operatorname{dim} \operatorname{Con}(M), l$ меняется от 1 до $L=[(n+1-m) / 3]$, а квадратные скобки обозначают иелую часть числа.

K сожалению, это утверждение оказалось неточным. Действительно, в размерности $n=4$ из этого утверждения следует, что степень подвижности равна 2 , тогда и только тогда, когда количество линейно независимых конциркулярных векторных полей равно 1. Другими словами, из приведенной теоремы вытекает, что не существует четырехмерных пространств недопускающих конциркулярные векторные поля, но допускающие степень подвижности $p \geqslant 2$. Однако такие метрики существуют. Примером такой метрики является ограничение стандартной метрики $d x_{1}^{2}+d x_{2}^{2}+\cdots+d x_{5}^{2}$ евклидового пространства $\mathbb{R}^{5}$, на стандартный эллипсоид

$$
\left\{\left(x_{1}, x_{2}, \ldots, x_{5}\right) \in \mathbb{R}^{5} \mid \frac{x_{1}^{2}}{a_{1}}+\frac{x_{2}^{2}}{a_{2}}+\cdots+\frac{x_{5}^{2}}{a_{5}}=1\right\}, \quad \text { где } \quad 0<a_{1}<a_{2}<\cdots<a_{5} .
$$

Как показано в [4]-[6], метрика эллипсоида допускает нетривиальную геодезическую эквивалентность. Поэтому степень подвижн ости этой метрики не меньше двух. С другой стороны, метрика эллипсоида не допускает конциркулярное векторное поле. Чтобы показать, что метрика эллипсоида не допускает конциркулярное векторное поле, мы

Работа первого и второго авторов выполнена при поддержке Немецкого научного общества (Deutsche Forschungsgemeinschaft, SPP 1154 - Globale Differentialgeometrie), работа третьего автора выполнена при поддержке Чешского грантового агенства (The Council of Czech Government, MSM 6198959214, MEB 040907). 
рассмотрим условия (2), представляющие собой систему дифференциальных уравнений на компоненты вектора $\varphi_{i}$. Продифференцировав ковариантно после альтернирования с учетом тождества Риччи найдем условия интегрируемости

$$
\varphi_{\alpha} R_{i j k}^{\alpha}=\rho_{k} g_{i j}-\rho_{j} g_{i k}
$$

этой системы. Здесь $R_{j k l}^{i}$ - тензор Римана, а $\rho_{i}:=\rho_{, i}$. Сворачивая уравнение (3), получаем

$$
\varphi^{\alpha} R_{\alpha i}=(n-1) \rho_{i}
$$

где $R_{i j}$ - тензор Риччи, $\varphi^{i}=\varphi_{\alpha} g^{\alpha i}, g^{i j}$ - элементы обратной матрицы метрики. Учитывая это, (3) примет вид

$$
\varphi^{\alpha} W_{\alpha i j}^{h}=0,
$$

где $W_{i j k}^{h}$ - тензор проективной кривизны Вейля. Прямым подсчетом убеждаемся в том, что тензор Вейля эллипсоида не допускает нетривиальных векторных полей, удовлетворяющих условиям (4).

Тем не менее, основная идея работы была правильной, используя эту идею мы получаем следующее утверждение

Теорема 2. Степень подвижности $n$-мерного $(n \geqslant 3)$ риманова пространства, отличного от пространства постоянной кривизнь, может принимать лишь следующие значения $p=m(m+1) / 2+l$, где $m=\operatorname{dim} \operatorname{Con}(M), l$ меняется от 1 до $L=[(n+1-m) / 3]$, квадратные скобки обозначают целую часть числа, или $p=1,2$.

Мы видим, что утверждение теоремы 2 отличается от утверждения теоремы 1 тем, что в теореме 2 степень подвижности, кроме прочих значений, может еще равняться 1 или $2 ; p \leqslant 2$ - особый случай, не связанный с количеством конциркулярных векторных полей.

Ошибка в доказательстве теоремы 1 как раз и связана со случаем $p \leqslant 2$. Доказывая теорему 1, автор неявно предполагает, что пространс тво является так называемым пространством $V_{n}(B)[7],[8]$. Однако не каждое пространство допускающее $p=2$ является пространством $V_{n}(B)$. Упомянутая выше метрика эллипсоида не является метрикой $V_{n}(B)$, так как $V_{n}(B)$ - метрика на замкнутом многообразии обязана быть метрикой пространства постоянной кривизны [9], [10], [11].

С другой стороны, как было показано [7], [8], каждая метрика при $p \geqslant 3$ является метрикой $V_{n}(B)$.

Таким образом, доказательство работы [3] корректно при дополнительном предположении $p>2$, поэтому, после добавления слов “или $p=1,2$ ", утверждение теоремы становится верным.

\section{СПИСОК ЦИТИРОВАННОЙ ЛИТЕРАТУРЫ}

[1] Н. С. Синюков, Геодезические отображения римановых пространств, Наука, М., 1979. [2] Й. Микеш, Геометрия - 2, Итоги науки и техн. Сер. Соврем. мат. и ее прил. Темат. обз., 11, ВИНИТИ, М., 2002, 121-162. [3] И. Г. Шандра, Матем. заметки, 68:4, (2000), 620-626. [4] V.S. Matveev, P. I. Topalov, Regul. Chaotic Dyn., 3:2, (1998), 30-45. [5] S. Tabachnikov, Comment. Math. Helv., 74:2, (1999), 306-321. [6] P. Topalov, Acta Appl. Math., 59:3, (1999), 271-298. [7] А.С. Солодовников, Тр. сем. по вект. и тенз. анализу, 11, Изд-во МГУ, М., 1961, 43-102. [8] В. А. Киосак, Й. Микеш, Геометрия погруженных многообразий, Изд-во МГПИ, М., 1986, 35-39. [9] V. S. Matveev, Singularity Theory and its Applications, Adv. Stud. Pure Math., 43, Math. Soc. Japan, Tokyo, 2006, 221-250. [10] V.S. Matveev, J. Differential Geom., 75:3, (2007), 459-502. [11] V. S. Matveev, Projective Lichnerowicz-Obata conjecture, arXiv: math.DG/0407337. 